\title{
Designing and Troubleshooting Immunopanning Protocols for Purifying Neural Cells
}

\author{
Ben A. Barres ${ }^{1}$ \\ Stanford University School of Medicine, Stanford, California 94305
}

Purifying and culturing cells from the central nervous system (CNS) has proved to be an incredibly powerful tool for dissecting fundamental neuron and glial properties, and especially powerful in understanding neuronal-glial interactions. In a series of detailed protocols, we have provided stepby-step instructions for purifying and culturing specific types of neurons, glia, and vascular cells from the CNS by immunopanning. This article discusses common pitfalls and errors as well as important design considerations for the immunopanning procedure.

The nervous system is the most complex organ in our body. In humans, it has been estimated to contain as many as one trillion cells, including neurons, glia, and vascular cells. Understanding the interactions between these cells and their networks is critical for understanding the development and function of our nervous system, as well as how these processes go awry in disease. How can we begin to understand and unravel such complexity? In other organs, such as the immune system, purification of cell types has provided a powerful way forward.

As a PhD student with Linda Chun and David Corey in the 1980s, I developed a method to purify retinal ganglion cells (RGCs) from the postnatal rat retina using immunopanning (Barres et al. 1988). In immunopanning, antibodies are adsorbed to the surface of a Petri dish, and cell suspensions are incubated on this dish to select for or deplete cell types of interest. In a typical immunopanning purification, a cell suspension from the retina or other nervous system region is prepared and then passed over several immunopanning dishes consecutively, with the first dish or two being used to deplete unwanted cell types, such as microglia, and the final dish being used to select the cell type of interest. In my own laboratory over the past 20 years, we have continued to use immunopanning to develop purification and culture methods for many other CNS cell types, including other neuronal and glial types as well as vascular cells. The talented young people who have used and developed these methods, all of whom are now in their own laboratories, have each authored protocols detailing the purification and culture methods for these cell types (see Related Information).

Using the methods described in these protocols, it is now possible to prepare serum-free cultures of prospectively isolated neurons and glia whose properties in culture very closely resemble their in vivo counterparts. This provides the opportunity to begin to do many types of studies to understand the fundamental properties of neurons and glia, as well as their interactions with each other and with

\footnotetext{
${ }^{1}$ Correspondence: barres@stanford.edu

(C) 2014 Cold Spring Harbor Laboratory Press

Cite this introduction as Cold Spring Harb Protoc; doi:10.1101/pdb.ip073999
} 
vascular cells. Below, troubleshooting information for immunopanning purification is provided, and considerations for the design of successful immunopanning protocols are discussed.

\section{COMMON ERRORS IN IMMUNOPANNING}

Purification of cells by panning is simple and provides high-yield, high-viability cells reliably, once you get the hang of it. Generally, the method takes practice, as every step must be performed correctly to have the cells still in good shape by the end of the procedure. Two of the most common errors that are made are (1) not using Worthington Biochemical as the source for papain and (2) not using appropriately prepared and stored trypsin for the removal of the purified cells from the final panning dish. (Trypsin will cleave itself over time if not stored at $-80^{\circ} \mathrm{C}$ - though genetically engineered preparations of trypsin are now available that are modified to prevent this.)

Panning is trivial, involving only three steps: (1) enzymatic preparation of a cell suspension, (2) passing this suspension over a series of antibody-coated dishes, and (3) removing the purified cells from the final dish. In general, the following guidelines should be followed: attempt to minimize the length of the entire procedure, avoid cooling mammalian neurons lower than room temperature even for only a few minutes, and never centrifuge enzymatically isolated neural cells in a solution containing less than $10 \%$ fetal calf serum (FCS) or $0.5 \%$ bovine serum albumin (BSA). This is particularly true if the cells have been twice exposed to enzyme - for instance, to papain for preparation of the initial cell suspension followed by trypsin to remove them from the dish. Centrifugation without sufficient protein in the solution after the trypsin step will cause death of most of the cells. In addition, avoid using bicarbonatebuffered solutions in the procedures, except with cells being stored in $\mathrm{CO}_{2}$ incubator.

When a low yield of cells is obtained from an immunopanning purification, it is critical to determine at what stage of the procedure the yield is low. If it is low after the initial preparation of the cell suspension, this suggests that the papain or DNase may not be active or used at sufficient concentration (or that magnesium has been omitted from the DNase solution, which needs to contain at least $100 \mu \mathrm{M} \mathrm{Mg}^{2+}$ ). On the other hand, if the yield is normal after preparation of the cell suspension but the density of cells looks low on the final panning dish, this suggests that the problem was with the preparation of that final dish. Essential questions would be was the secondary antibody (the initial antibody placed on the dish) the right isotype, was it applied in a buffer at $\mathrm{pH} 9.5$, is the dish a Petri dish and not a tissue culture dish, and was the primary antibody used at too high or too low a concentration? If the density of cells looks good on the final dish, but the final yield after the cells have been trypsinized is low, then it may be that the trypsin was not sufficiently active, or the cells were not centrifuged in a solution containing sufficient protein concentration. In addition, temperature control is critical for good viability. As has already been mentioned, chilling of mammalian neurons, even briefly, can lead to very low viability. Another, easily overlooked problem is that old hot water baths often become unstable and undergo constant temperature oscillations that can lead to incubation temperatures that are too high. Even half a degree or one degree too high can lead to low viability and cell death.

If the viability of the purified cells is low, it is similarly critical to determine when the viability became low. If the viability is $<85 \%$ after preparation of the tissue cell suspension (before panning), typically very few of these cells will survive in culture. If the viability is low immediately after removing the cells from the final panning dish, this suggests either that the trypsin was not active or that the cell surface antigen was not trypsin-sensitive; typically, the yield would be very low in these cases as well. If the yield and viability are fine after panning but viability is poor after a few days of culture, then there is almost certainly a problem with the culture conditions: The medium or growth factors could be expired or defective, the substrate may not be optimal, the glass coverslips might not have been 
B.A. Barres

cleaned sufficiently, or the tissue culture plastic may be leaching a toxic plastic stabilizing resin (these resins can also leak from certain brands of plastic syringes). A very common problem is that the $\mathrm{CO}_{2}$ concentration in the incubator is not correct, even though the incubator $\mathrm{CO}_{2}$ meter claims that it is. Incubators need to be calibrated frequently.

\section{CULTURE MEDIA}

The composition of serum-free media is critical to good viability. In addition to having appropriate growth factors or other signals necessary to prevent apoptosis, medium must be the correct $\mathrm{pH}$ and osmolarity. Common base medium solutions used for culture of neural cells vary dramatically in their osmolarity. For instance, Dulbecco's modified Eagle's medium (DMEM) has an osmolarity of 330 mOsm, whereas Neurobasal has an osmolarity of only $\sim 220$. (Normal cerebrospinal fluid is more typically $\sim 290-300 \mathrm{mOsm}$.) It is important to keep in mind that Neurobasal is an extremely minimal medium, with fewer substances in lower concentrations (such as amino acids) when compared to DMEM. In our experience, it is therefore much easier for Neurobasal to become inactive. To avoid this potential problem, we sometimes use DMEM as the base medium, but dilute it by $\sim 25 \%$ to bring it down to the osmolarity of Neurobasal. Alternatively, DMEM and Neurobasal can be used as a 50:50 mixture, which also brings osmolarity closer to normal. The osmolarity of the final medium is increased, of course, by the addition of the many additives such as glutamine, penicillin/streptomycin, etc.- - often by as much as another $35 \mathrm{mOsm}$.

Another very important consideration in achieving good viability is the quality of the serum-free additives that are included in the final culture medium, such as the Bottenstein-Sato serum-free additive, B-27, or NS21. In our experience, commercially obtained B-27 (which is tested by the company only for hippocampal neurons) has been associated in recent years with toxicity for at least some or all of the cell types described in our protocols. For this reason, we have moved to the NS21 additive, which we formulate ourselves (Chen et al. 2008). The Bottenstein-Sato reagent is made differently depending upon whether it is commercially obtained or homemade. The reagents in this additive typically include transferrin, sodium selenite, putrescine, progesterone, BSA, and, optionally, thyroxine and tri-iodothyronine. In our experience, the transferrin and selenite are the most critical, as they are crucial for both survival and proliferation of cells. Because transferrin is expensive, commercial sources typically have far lower concentrations than are optimal. When using BSA with primary cells, it is important to use a crystalline, relatively pure preparation to avoid toxic contaminants.

\section{TIPS FOR DESIGNING NEW PANNING PROTOCOLS}

General points that may be helpful in troubleshooting and designing new immunopanning protocols are described in the following section. In addition, a list of immunolabeling antibodies useful for identifying cell types is provided in Table 1.

\section{Enzymatic Preparation}

For panning, it is preferable to use antibodies that recognize surface antigens that are not cleaved off by the papain or trypsin used to prepare the cell suspension. For this reason, antibodies to glycolipids (e.g., GC) or gangliosides (e.g., A2B5) are ideal. In some cases when the antigen is a protein, the enzymes used do not completely cleave the antigen of interest and so enzymes can still be used (e.g., RAN-2 and Thy1). In many cases, however, the enzymes completely destroy the antigen. It is still possible to pan using antibodies to this antigen, if you allow the cell suspension to recover at $37^{\circ} \mathrm{C}$ for $30 \mathrm{~min}$ to $1 \mathrm{~h}$. This allows protein already in the secretory pathway to be transferred to the cell membrane. If you do this, the cell suspension should be pipetted and passed through a nylon mesh filter (Nitex) before it is added to the panning dish, so that any cell clumps that have aggregated during 
TABLE 1. Immunolabeling antibodies for identifying cell types

\begin{tabular}{llll}
\hline Cell type & Antigen & Antibody source & Dilution \\
\hline \multirow{2}{*}{ Astrocytes } & GFAP (anti-glial fibrillary acidic protein) & Sigma-Aldrich G3893 & $1: 2000$ \\
& Anti-AQP4 (aquaporin 4) & Sigma-Aldrich A5971 & $1: 1000$ \\
\multirow{3}{*}{ Endocytes } & GLT-1 (glutamate transporter 1; EAAT2) & Abcam ab41621 & $1: 5000$ \\
& CD31 (PECAM-1) & BD Pharmingen 553370 & $1: 500$ \\
Pericytes & Occludin & Invitrogen 71-1500 & $1: 250$ \\
& NG2 (chondroitin sulfate proteoglycan) & Millipore AB5320 & $1: 400$ \\
OPC & PDGFR $\beta$ & R\&D Systems AF385, AF1042 & $1: 500$ \\
& NG2 & Millipore AB5320 & $1: 400$ \\
& CNPase (2', 3'-Cyclic nucleotide 3'-phosphodiesterase) & Millipore MAB326 & $1: 100$ \\
& PDGFR $\alpha$ & BD Pharmingen 558774 & $1: 5000$ \\
OL & O4 & Millipore MAB345 & $1: 100$ \\
& MBP (myelin basic protein) & Abcam ab7349 & $1: 100$ \\
& PLP (proteolipid protein) & Millipore MAB388 & $1: 400$ \\
& MAG (myelin associated glycoprotein) & Millipore MAB1567 & $1: 50$ \\
\hline
\end{tabular}

the incubation period are eliminated. Clumps are a major threat to purity when they adhere to the final panning dish, as they can bring down unwanted contaminating cell types.

\section{Panning}

Always pan at room temperature. Never pan at $37^{\circ} \mathrm{C}$, because this allows activation of cell adhesion mechanisms and lets unwanted contaminating cell types adhere to panning dishes. Always use at least two panning dishes. The first should be coated with an antibody to deplete an unwanted contaminating cell type or with an irrelevant antibody to ensure that microglia/macrophages are depleted. Microglia/macrophages represent a high percentage of cells in the developing nervous system (e.g., in the developing optic nerve, they make up $\sim 6 \%$ of cells) and stick via their Fc receptors to the first antibody-coated dish they contact. They will not be successfully eliminated by panning on a dish that is not antibody-coated. An alternative for removing microglia/macrophages is to just coat the first dish with the lectin BSLI (Bandeiraea simplicifolia Lectin I; this lectin also binds to brain endothelial cells) or the CD45 monoclonal antibody.

Regarding the antibodies used to coat panning dishes, the first antibody (the goat anti-rabbit or anti-mouse) should always be affinity purified. That is, the main protein in this solution should be the antibody of interest; otherwise, irrelevant proteins such as BSA will hog most of the binding sites on the Petri dishes. This is why monoclonal supernatants, for instance, which generally contain $10 \%$ FCS, cannot be put straight onto the dish without precoating with an affinity purified anti-mouse antibody. If you happen to have an affinity-purified antibody (or a lectin) to the cell surface antigen of interest, then it can be put straight onto the dish (in a pH 9.5 solution, which is critical for good absorbance of the protein to the plastic).

In addition, the second antibody (the one that actually binds to the cell surface antigen of interest; see Table 2) should be a monoclonal supernatant whenever possible. The reason for this is that the only mouse antibody type in the supernatant is the one of interest. If you use a polyclonal antiserum, then there will be many irrelevant antibodies that will hog (anti-rabbit) sites on the panning dish. Similarly, many antibodies secreted into ascites are irrelevant host-derived antibodies and will also hog (anti-mouse) sites on the panning dish. In some cases, ascites from IgM-secreting hybridoma cells can be used as long as an anti-mouse IgM, $\mu$-chain-specific antibody is used to first coat the dish, as there do not seem to be many irrelevant IgM antibodies in ascites.

If there is no alternative and a polyclonal antiserum or ascites must be used, then it is still possible to use this antibody for panning. In this case, however, the panning dish must be coated with only one antibody (the anti-rabbit or anti-mouse antibody) and the cell suspension should be incubated with the rabbit or mouse antibody directly. In this way, the wanted antibodies will bind to the cell surface and the unwanted ones can be eliminated by centrifuging the cell suspension and discarding the supernatant (and preferably washing the suspension one more time). 
TABLE 2. Primary antibodies for immunopanning purification of neural cells

\begin{tabular}{ll}
\hline Antibody & Source \\
\hline Mouse anti-p75 NGF receptor antibody & Abcam ab6172 \\
Mouse anti-CTB (clone 3D11) & Meridian Life Science C86204M \\
Rat anti-mouse CD140a (PDGFR $\alpha$ ) & BD Pharmingen 558774 \\
Mouse anti-rat CD31 & Fitzgerald 10R-CD31gRT \\
Mouse anti-rat CD45 & AbD Serotec MCA589 \\
Rat anti-mouse CD31 & BD Pharmingen 553370 \\
Rat anti-mouse CD45 & BD Pharmingen Rat anti-ms550539 (Clone 30F-11) \\
C5 hybridoma supernatant & Mouse \\
Goat anti-human PDGFR $\beta$ & R\&D Systems AF385 \\
Goat-anti mouse PDGFR $\beta$ & R\&D Systems AF1042 \\
T11D7 supernatant & Mouse \\
Rabbit anti-rat antimacrophage sera & Accurate Chemical AIAD51240 \\
Ran-2 hybridoma supernatant & Mouse \\
Anti-O1 & Millipore MAB344 \\
Anti-O4 & Millipore MAB345
\end{tabular}

Removing Cells from the Final Dish

The most delicate step in panning is removing the tightly adhering purified cells from the panning dish. Although saturating amount of antibody can be used on the first panning dishes for depletion of contaminants from the cell suspension, the concentration of the antibody on the final panning dish is crucial and must be titrated carefully. If the cells are too tightly bound they will be difficult to release viably; if they are too loosely bound they will wash off and the yield will be low. This is particularly crucial if the antigen is not destroyed by trypsin, as antibodies are not well cleaved by trypsin. In general, an optimal concentration of antibody causes about half of the adherent cells to be phase dark and flat (tightly adherent) and the other half to be phase bright and roundish (less tightly adherent). This can be determined by a titration experiment in which the yield of viable cells is optimized; typically, supernatants on the final dish are diluted 1:20 and ascites $\sim 1: 2000$.

In general, if the antigen used to purify cells on the final panning dish is not trypsin-sensitive, then the antibody used needs to be an IgM rather than an IgG. The IgG-bound cells are difficult to release viably, perhaps because of high affinity for the antigen. If the only antibody available is an IgG and the antigen is not trypsin-sensitive, it is still possible to purify the cells. In this case, the final dish must be coated with either protein A or protein G (or a mixture of both) rather than an anti-mouse IgG antibody. Protein A and protein G are easily cleaved by trypsin and the cells are easily released.

Keeping the above considerations in mind, it should be straightforward to achieve purified cells in high yield with excellent viability and to design new immunopanning procedures.

\section{RELATED INFORMATION}

Step-by-step instructions for purifying and culturing specific types of neurons, glia, and vascular cells from the central nervous system by immunopanning are provided in the following protocols: Purification of Oligodendrocyte Precursor Cells from Rat Cortices by Immunopanning (Dugas and Emery 2013), Purification of Oligodendrocyte Precursor Cells from Mouse Cortices by Immunopanning (Emery and Dugas 2013), Purification and Culture of Spinal Motor Neurons from Rat Embryos (Graber and Harris 2013), Purification of Rat and Mouse Astrocytes by Immunopanning (Foo 2013), Purification and Culture of Retinal Ganglion Cells from Rodents (Winzeler and Wang 2013), Immunopanning of Retrograde-Labeled Corticospinal Motor Neurons from Early Postnatal Rodents (Mandemakers 2014), Purification of Dorsal Root Ganglion Neurons from Rat by Immunopanning (Zuchero 2014), Purification of Pericytes from Rodent Optic Nerve by Immunopanning (Zhou et al. 2014a), Purification of Endothelial Cells from Rodent Brain by Immunopanning (Zhou 
et al. 2014b), Myelinating Cocultures of Rat Retinal Ganglion Cell Reaggregates and Optic Nerve Oligodendrocyte Precursor Cells (Watkins and Scholze 2014), and Purification of Schwann cells from the Neonatal and Injured Adult Mouse Peripheral Nerve (Lutz 2014).

\section{REFERENCES}

Barres BA, Silverstein BE, Corey DP, Chun LL. 1988. Immunological, morphological, and electrophysiological variation among retinal ganglion cells purified by panning. Neuron 1: 791-803.

Chen Y, Stevens B, Chang J, Milbrandt J, Barres BA, Hell JW. 2008. NS21: Re-defined and modified supplement B27 for neuronal cultures. $J$ Neurosci Meth 171: 239-247.

Dugas JC, Emery B. 2013. Purification of oligodendrocyte precursor cells from rat cortices by immunopanning. Cold Spring Harb Protoc doi: $10.1101 /$ pdb.prot070862.

Emery B, Dugas JC. 2013. Purification of oligodendrocyte precursor cells from mouse cortices by immunopanning. Cold Spring Harb Protoc doi: $10.1101 /$ pdb.prot073973.

Foo LC. 2013. Purification of rat and mouse astrocytes by immunopanning. Cold Spring Harb Protoc doi: 10.1101/pdb.prot074211.

Graber DJ, Harris BT. 2013. Purification and culture of spinal motor neurons from rat embryos. Cold Spring Harb Protoc doi: 10.1101/pdb. prot074161.

Lutz AB. 2014. Purification of Schwann cells from the neonatal and injured adult mouse peripheral nerve. Cold Spring Harb Protoc doi: 10.1101/ pdb.prot074989.
Mandemakers W. 2014. Immunopanning of retrograde-labeled corticospinal motor neurons from early postnatal rodents. Cold Spring Harb Protoc doi: 10.1101/pdb.prot074930.

Watkins TA, Scholze AR. 2014. Myelinating cocultures of rat retinal ganglion cell reaggregates and optic nerve oligodendrocyte precursor cells. Cold Spring Harb Protoc doi: 10.1101/pdb.prot074971.

Winzeler A, Wang JT. 2013. Purification and culture of retinal ganglion cells from rodents. Cold Spring Harb Protoc doi: 10.1101/pdb. prot074906.

Zhou L, Sohet F, Daneman R. 2014a. Purification of pericytes from rodent optic nerve by immunopanning. Cold Spring Harb Protoc doi: 10.1101/ pdb.prot074955.

Zhou L, Sohet F, Daneman R. 2014b. Purification of endothelial cells from rodent brain by immunopanning. Cold Spring Harb Protoc doi: 10.1101/ pdb.prot074963.

Zuchero JB. 2014. Purification of dorsal root ganglion neurons from rat by immunopanning. Cold Spring Harb Protoc doi: 10.1101/pdb. prot074948. 


\title{
Designing and Troubleshooting Immunopanning Protocols for Purifying Neural Cells
}

\author{
Ben A. Barres
}

Cold Spring Harb Protoc; doi: 10.1101/pdb.ip073999

\begin{tabular}{|c|c|}
\hline $\begin{array}{r}\text { Email Alerting } \\
\text { Service }\end{array}$ & Receive free email alerts when new articles cite this article - click here. \\
\hline $\begin{array}{l}\text { Subject } \\
\text { Categories }\end{array}$ & $\begin{array}{l}\text { Browse articles on similar topics from Cold Spring Harbor Protocols. } \\
\text { Immunoaffinity Purification ( } 49 \text { articles) } \\
\text { Immunoseparation ( } 31 \text { articles) } \\
\text { Mouse ( } 437 \text { articles) } \\
\text { Neural Cell Culture ( } 57 \text { articles) } \\
\text { Neuroscience, general ( } 357 \text { articles) } \\
\text { Other Laboratory Organisms (68 articles) }\end{array}$ \\
\hline
\end{tabular}

